Rev. Biol. Trop., 46(2): 277-284, 1998

\title{
Composición y distribución de la ictiofauna en la Laguna del Mar Muerto, Pacífico mexicano
}

\author{
M. Tapia-García 1, C. Suárez Núñez 1, G. Cerdenares L. de Guevara 1, M. C. Macuitl Montes ${ }^{1}$, \\ M. C. García Abad 1
}

1 Departamento de Hidrobiología, D.C.B.S. Universidad Autónoma Metropolitana-Iztapalapa, Apartado Postal 55-535, México 09340. e-mail mtg@xanum.uam.mx

Recibido 2-V-1997. Corregido 31-X-1997. Aceptado 14-I-1998.

\begin{abstract}
Fish composition was sampled in Mar Muerto Lagoon (Mexico) between 1991 and 1993. A total of 27971 fish with a weight of $120.9 \mathrm{~kg}$ were collected. The total sample consists of 29 families, 45 genera and 66 species. The highest number of species is in the area with strong marine influence (near Tonala Inlet), abundant coastal vegetation and fluvial discharge. The lowest number of species is in the inner part of the lagoon, which is hipersaline in the dry season and of low salinity in the rainy season. The species with broad distribution are Diapterus peruvianus, Lile stolifera, Anchoa lucida, Anchoa mundeola, Mugil curema, Atherinella guatemalensis and Anchovia macrolepidota.
\end{abstract}

Key words: Dominance, estuarine system, fish distribution, Gulf of Tehuantepec, Mar Muerto Lagoon, Mexico.

En el sur del Pacífico mexicano se localiza un gran número de sistemas lagunares-estuarinos, donde la actividad pesquera artesanal es intensa y dirigida principalmente al camarón (Penaeus). Esto, aunado a la expansión industrial, principalmente la del petróleo, determina al Golfo de Tehuantepec, y por consiguiente a la Laguna del Mar Muerto, como área prioritaria en cuanto a la necesidad de estudios de evaluación ecológica de sus recursos. A pesar de que los sistemas costeros de la región sufren una intensa explotación pesquera, no existen estudios donde se evalúen sus recursos bióticos tanto en composición de especies, como en su abundancia. La Laguna del Mar Muerto es un sistema poco estudiado que además presenta una situación geográfica política que agudiza la problemática, ya que, el extre- mo oeste de la laguna forma parte del estado de Oaxaca y la parte este de Chiapas.

Con relación a las comunidades de peces en otras lagunas costeras de la región se pueden mencionar los trabajos de Castro-Aguirre et al. (1974), Chávez (1979) y Castro-Aguirre (1982) sobre el complejo lagunar Oriental-Occidental. El único antecedente existente sobre la ictiofauna de la laguna es el de Alvarez del Villar y Díaz Pardo (1973). Esto hace evidente la casi nula información existente sobre los recursos ícticos de estos sistemas en el área. Por esta razón, el objetivo del presente trabajo fue caracterizar la composición de especies de peces y su distribución en la Laguna del Mar Muerto como un paso preliminar al conocimiento ecológico de la región. 


\section{MATERIALES Y MÉTODOS}

La Laguna del Mar Muerto, se localiza en la parte norte del Golfo de Tehuantepec en el Pacífico Sur mexicano, formando parte de los estados de Oaxaca y Chiapas (Fig. 1). Tiene una longitud aproximada de $12 \mathrm{~km}$ en su parte más ancha y $60 \mathrm{~km}$ de largo y se conecta con la línea de costa del Océano Pacífico por la Boca de Tonalá (Cervantes-Castro 1969). Se observan dos épocas climáticas bien definidas correspondientes a la época de sequía (noviembre a abril) y a la época de lluvias (mayo a octubre) (Anónimo 1980). Hacia la parte sureste prevalecen condiciones marinas, que no tienen variación por el aporte constante de agua de mar a través de la Boca de Tonalá, y en el área central el intercambio es limitado provocando una variación estacional. El área noroeste es muy somera y presenta grandes variaciones estacionales, lo que determina que sea un sistema de condiciones eurihalinas. Desem- bocan un gran número de ríos de escasa dimensión los que se mantienen secos durante la época de sequía, de los cuales el de mayor importancia es el río Tapanatepec que desemboca en la región denominada "El Escopetazo" (Alvarez del Villar y Díaz Pardo 1973).

En la Laguna del Mar Muerto se realizaron 13 salidas de campo de marzo a diciembre en un periodo de tres años (1991-1993), cubriendo un total de 61 estaciones de muestreo (Fig. 1). Esta red de estaciones permitió caracterizar áreas contrastantes dentro de la Laguna del Mar Muerto: ambientes de baja salinidad, hipersalinidad y marinos-. En 33 estaciones las colectas se hicieron con una red de arrastre de prueba camaronera de $5 \mathrm{~m}$ de largo, $2.4 \mathrm{~m}$ de abertura de trabajo y luz de malla de $3 / 4$ ", a una velocidad promedio de 2 nudos y una duración de 15 minutos. Las 28 colectas restantes se realizaron con una red tipo chinchorro playero de $20 \mathrm{~m}$ de largo y una luz de malla de $3 / 4$ ". Las

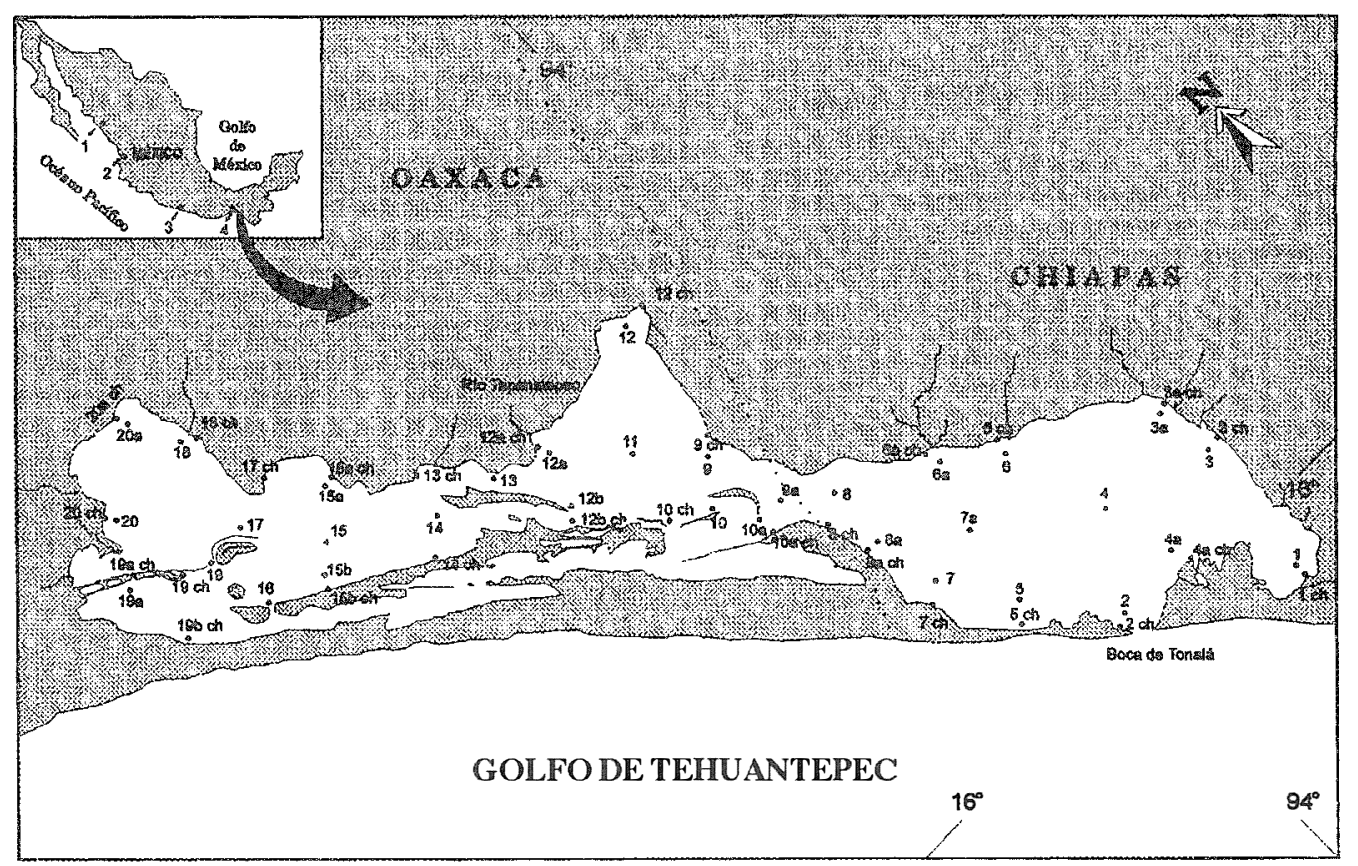

Fig. 1 Localización del área de estudio, así como las estaciones de muestreo. El número 1 corresponde a la ubicación del sistema Huizache-Caimanero, Sinaloa; el 2 al sistema Teacapán-Agua Brava, Nayarit, el 3 a las lagunas de Guerrero, y el 4 a las lagunas Oriental-Occidental, Oaxaca. 
muestras obtenidas fueron fijadas en formaldehído al $10 \%$ neutralizado con borato de sodio y empacadas en bolsas de plástico para su posterior procesamiento en el laboratorio.

En el laboratorio, los peces e invertebrados fueron separados, lavados y colocados en alcohol etílico al $70 \%$ para su posterior identificación taxonómica empleando la literatura específica (Jordan y Evermann 1896-1900, Meek 1902-1904, Meek y Hildebrand 19231928, Castro-Aguirre 1978, Amezcua-Linares 1990, entre otros).

\section{RESULTADOS Y DISCUSION}

Composición taxonómica: Se determinó un total de 66 especies, 45 géneros y 29 familias que se muestran en la siguiente lista sistemática ordenada de acuerdo a Greenwood et al. (1966) modificada por Nelson (1994):

Lista Sistemática de Feces de la Laguna del Mar Muerto, Oaxaca-Chiapas.

\section{Familia Elopidae}

Elops affinis Reagan, 1909

Familia Albulidae

Albula vulpes (Linnaeus, 1758)

Familia Chanidae

Chanos chanos (Forksal, 1775)

Familia Clupeidae

Opisthonema libertate (Günther, 1867)

Opisthonema medirastre Berry y Barret, 1964

Lile stolifera (Jordan y Gilbert, 1881)

Familia Engraulididae

Anchoa argentivittata (Ragan, 1904)

Anchoa curta ( Jordan y Gilbert, 1882)

Anchoa ischana (Jordan y Gilbert, 1882)

Anchoa lucida (Jordan y Gilbert, 1882)

Anchoa mundeola (Gilbert y Pierson, 1898)

Anchovia macrolepidota (Kner y Steindachner, 1865)

Familia Ariidae

Cathorops liropus (Bristol, 1906)

Galeichthys caerulescens Günther, 1864
Familia Batrachoididae

Batrachoides waltersi Collette y Russo, 1981

Familia Poeciliidae

Poecilia sphenops Cuvier y Valenciennes, 1836

Poeciliopsis fasciata Meek, 1904

Familia Anablepidae

Anableps dovii Gill, 1861

Familia Exocoetidae

Hyporhamphus rosae (Jordan y Gilbert, 1880)

Hyporhamphus unifasciatus (Ranzani, 1842)

Familia Belonidae

Tylosurus exilis (Girard, 1854)

Familia Atherinidae

Atherinella guatemalensis (Günther, 1864)

Familia Centropomidae

Centropomus robalito Jordan y Gilbert, 1882

Centropomus nigrescens Günther, 1869

Familia Serranidae

Epinephelus multiguttatus (Günther, 1866)

Familia Carangidae

Caranx caninus Günther, 1869

Oligoplites altus (Günther, 1868)

Oligoplites mundus Jordan y Starks, 1898

Oligoplites refulgens Gilbert y Starks, 1904

Oligoplites saurus (Bloch y Schneider, 1801)

Selene oerstedii Lütken, 1880

Familia Lutjanidae

Lutjanus argentiventris (Peters, 1869)

Lutjanus colorado Jordan y Gilbert, 1882

Lutjanus guttatus (Steindachner, 1869)

Familia Gerreidae

Diapterus aureolus (Jordan y Gilbert, 1881)

Diapterus peruvianus (Cuvier y Valenciennes, 1830)

Eucinostomus currani Yánez-Arancibia, 1980

Eucinostomus dowi (Gill, 1863)

Eucinostomus gracilis (Gill, 1862)

Gerres cinereus (Walbaum, 1792)

Familia Haemulidae

Anisotremus dovii (Günther, 1864)

Anisotremus pacifici (Günter, 1864)

Pomadasys axillaris (Steindachner, 1869)

Pomadasys leuciscus (Günther, 1864)

Pomadasys macracanthus (Günther, 1864)

Familia Sciaenidae

Cynoscion stolzmanni (Steindachner, 1879)

Micropogonias altipinnis (Günter, 1864) 
Familia Ephippidae

Chaetodipterus zonatus (Girard, 1858)

Familia Chaetodontidae

Chaetodon humeralis Günther, 1860

Familia Cichlidae

Cichlasoma octofasciatum (Regan, 1906)

Familia Mugilidae

Mugil cephalus Linnaeus, 1758

Mugil curema Valenciennes, 1836

Familia Polynemidae

Polydactylus approximans (Lay y Bennet, 1839)

Familia Gobiidae

Gobionellus microdon (Gilbert, 1891)

Gobionellus sagittula (Günther, 1861)

Microgobius miraflorensis Gilbert y Starks, 1904

Familia Eleotridae

Dormitator latifrons (Richardson, 1844)

Gobiomorus maculatus (Günther, 1859)

Familia Bothidae

Citharichthys gilberti Jenkins y Evermann, 1889

Cyclopsetta panamensis (Steindachner, 1875)

Etropus crossotus Jordan y Gilbert, 1882

Syacium ovale (Günther, 1864)

Familia Soleidae

Achirus mazatlanus (Steindachner, 1869)

Achirus zebrinus Clark, 1936

Familia Tetraodontidae

Sphoeroides annulatus (Jenyns, 1843)

Arothron hispidus (Linnaeus, 1758)

Distribución de la ictiofauna: Las especies determinadas aportaron un total de 27971 individuos con un peso de $120.9 \mathrm{~kg}$. De las especies colectadas solo fueron accesibles a la red tipo chinchorro 54 especies y a la red de arrastre 57 especies, siendo comunes para ambas artes de pesca el $69 \%$ (Cuadro 1 y 2). Al respecto, Alvarez del Villar y Díaz Pardo (1973), registraron un total de 36 especies en la Laguna del Mar Muerto, incrementándose en gran medida con el presente trabajo el número de especies registradas. El número de especies registradas es alto comparado con los informados para otros sistemas lagunares-estuarinos del Pacífico mexicano. Al respecto, Yáñez-
Arancibia (1978) informa para las lagunas del estado de Guerrero un total de 43 especies en la Laguna de Nuxco, 25 especies en la Laguna Salinas del Cuajo, 25 en la Laguna de Coyuca, 16 especies en la Laguna de Mitla, 33 especies en la Laguna de Tecomate, 39 especies en la Laguna Salinas de Apozahualco y 85 especies en la Laguna de Chautengo. Alvarez Rubio et al. (1986) informan un total de 76 especies en el sistema lagunar Teacapán-Agua Brava, Nayarit, mientras que para el sistema HuizacheCaimanero, Sinaloa, Amezcua-Linares (1977) informa 60 especies. En las lagunas OrientalOccidental adyacentes a la Laguna del Mar Muerto Castro-Aguirre et al. (1974), Chávez (1979) y Castro Aguirre (1982) informan un total de 72 especies (Fig. 1). Como se puede observar, el número de especies en sistemas lagunares-estuarinos del Pacífico mexicano es bajo, sobresaliendo en número de especies solo algunos sistemas que presentan valores semejantes a la Laguna del Mar Muerto.

En relación con las características contrastantes de la laguna con hipersalinidad en su parte mas interna en la época de secas y baja salinidad en la época de lluvias y con menor variación de salinidad hacia la Boca de Tonalá, de características marinas (Álvarez del Villar y Díaz Pardo 1973), la menor abundancia relativa de especies se presenta hacia la parte más interna también caracterizada por escasa vegetación costera y la mayor abundancia de especies en el extremo sureste en el área de influencia marina y con mayor vegetación costera. $\mathrm{Al}$ respecto, en estaciones donde hay tránsito de especies la abundancia de éstas es baja, como en el caso de las estaciones que se localizan en la barra de Tonalá o cercanas a ella (estaciones $2,4,4$ y 5). De las 66 especies registradas algunas presentan amplia distribución en la laguna reflejo de su dominancia, entre estas especies se encuentran $D$. peruvianus, $L$. stolifera, A. mundeola, A. lucida, y C. robalito. También fueron dominantes en algunos meses $A$. macrolepidota, A. guatemalensis, P. fasciata y $P$. sphenops. Algunas se distribuyen preferentemente hacia la parte más interna de la laguna 
CUADRO 1

Distribución por estación de las especies colectadas con red tipo chinchorro.

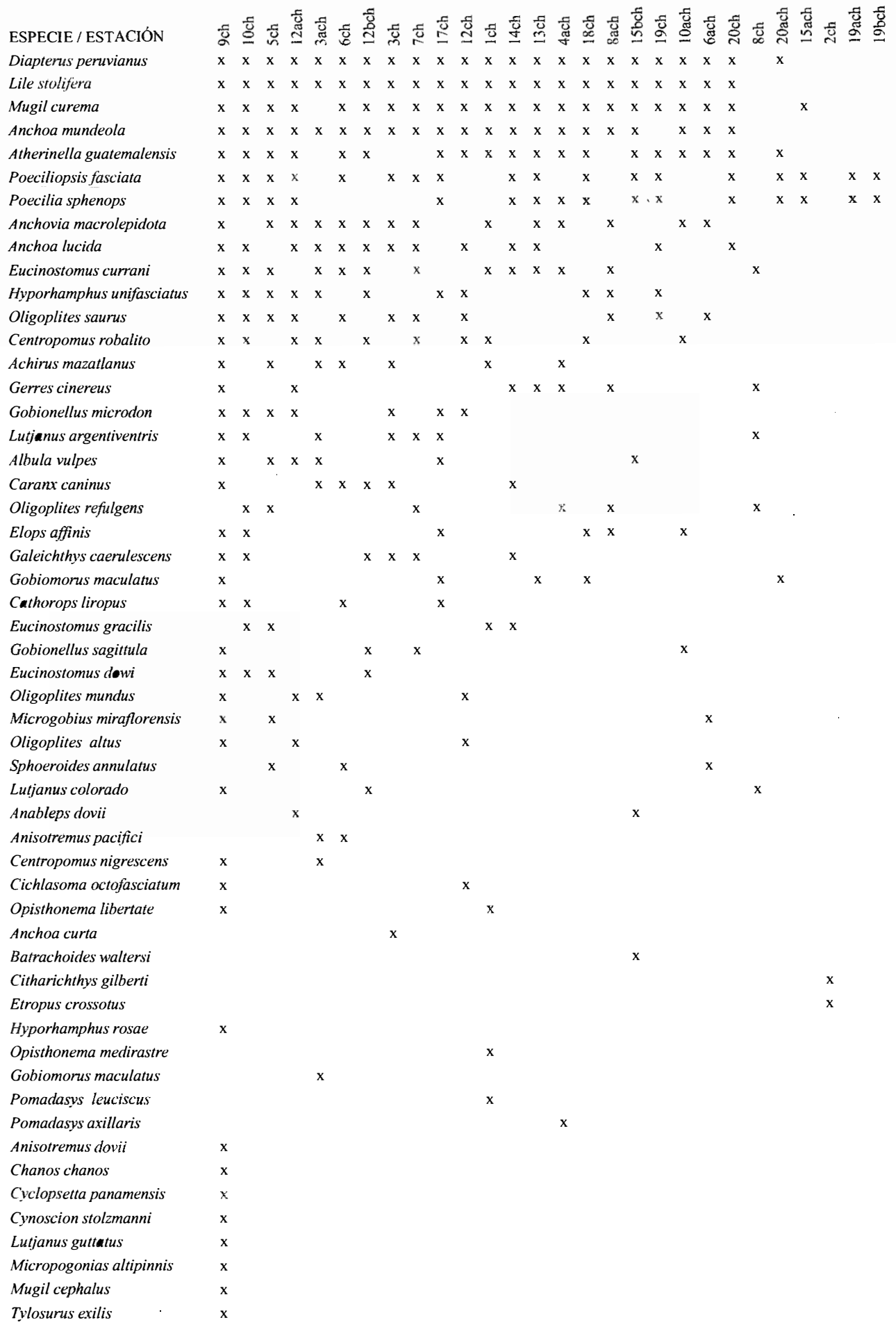


CUADRO 2

Distribución por estación de las especies colectadas con red de arrastre.

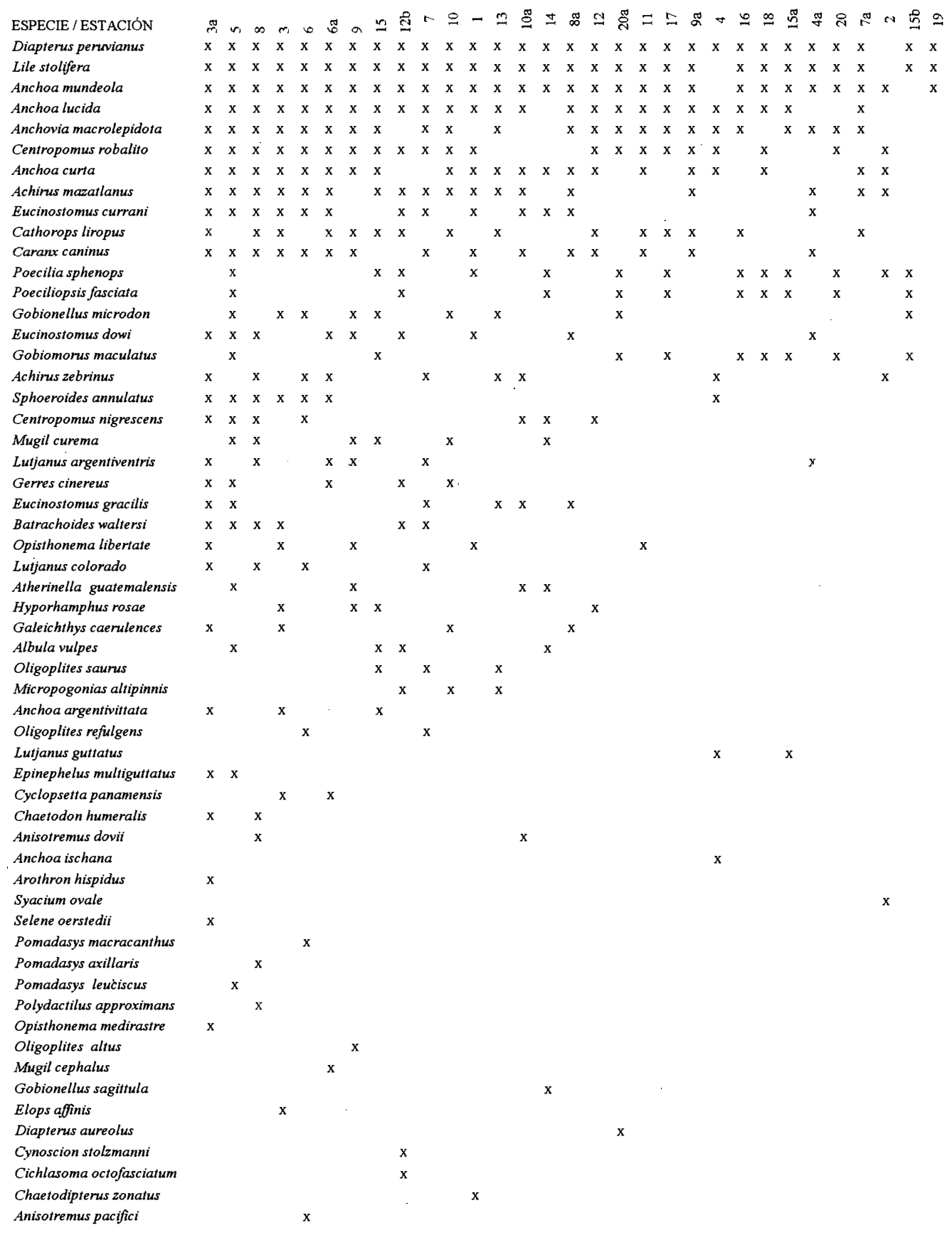


como es el caso de A. guatemalensis, $P$. fasciata y $P$. sphenops, y otras se distribuyen hacia el área de influencia marina, como son $D$. peruvianus, L. stolifera, A. mundeola y A. lucida. De las especies informadas por Tapia García et al. (1994) para la plataforma continental se observa que algunas especies de la Laguna del Mar Muerto también se distribuyen en la plataforma, lo que puede indicar una dependencia estuarina de esas especies. Estas especies son el $61 \%$ entre las que sobresalen por su amplia distribución D. peruvianus, A. mundeola, A. lucida, $C$. robalito y $M$. curema.

Asimismo, algunas especies se distribuyen solamente en ecosistemas lagunares estuarinos, lo que indica que son residentes permanentes en la Laguna del Mar Muerto, representando éstas el $37 \%$ de la composición de especies total y las más conspicuas por su amplia distribución son $L$. stolifera, $P$. sphenops, $P$. fasciata, $A$. guatemalensis e $H$. unifasciatus.

Los resultados obtenidos resaltan la importancia ecológica de la Laguna del Mar Muerto en la región, ya que además de constituir una área de alimentación, protección y crianza para el camarón en su etapa de postlarva y juvenil que es la principal actividad pesquera de la región, también un gran número de especies de peces dependen de este ecosistema de forma cíclica u ocasionalmente para completar sus ciclos biológicos. La importancia de estos ecosistemas lagunares-estuarinos en la dinámica ecológica de los ecosistemas adyacentes ya ha sido señalada por diversos autores, como lo menciona Yáñez-Arancibia (1986).

\section{AGRADECIMIENTOS}

El presente trabajo fue financiado por el proyecto de investigación "Composición, distribución y abundancia de la comunidad de peces de la Laguna del mar Muerto, OaxacaChiapas", UAM-I/CBS. Un agradecimiento especial a J. Alejandro Gamboa Contreras por su colaboración en la recolección. Asimismo, se agradece el apoyo brindado a la Cooperativa Pesquera "Progresistas del Mar", del poblado de la Gloria, Chiapas, en especial a sus dirigentes Apolinar López, Armando Torres, José Ramírez y Alberto Marroquí.

\section{RESUMEN}

La Laguna del Mar Muerto, Oaxaca-Chiapas fue muestreada entre 1991 y 1993 con el objetivo de caracterizar la ictiofauna en cuanto a su composición y abundancia. Se colectaron un total de 27971 peces que aportaron $120.9 \mathrm{~kg}$. La ictiofauna de la región está caracterizada por 29 familias, 45 géneros y 66 especies. El mayor número de especies se presenta en la parte sureste de la laguna (cerca de la Boca de Tonalá), área con mayor influencia marina, abundante vegetación costera y mayor descarga fluvial. El menor número de especies se presenta en la parte más interna de la laguna, caracterizada por hipersalinidad en la época de secas y baja salinidad en la época de lluvias. Las especies que presentan amplia distribución son Diapterus peruvianus, Lile stolifera, Anchoa lucida, Anchoa mundeola, Mugil curema, Atherinella guatemalensis y Anchovia macrolepidota.

\section{REFERENCIAS}

Alvarez del Villar, J \& E. Díaz Pardo. 1973. Estudio bioecológico del Mar Muerto, Oaxaca. Instituto Politécnico Nacional, Escuela Nacional de Ciencias Biológicas, México. 85 p.

Alvarez Rubio, M., F. Amezcua-Linares \& A. YáñezArancibia. 1986. Ecología y estructura de las comunidades de peces en el sistema lagunar TeacapánAgua Brava, Nayarit, México. An. Inst. Cien. del Mar y Limnol. Univ. Nal. Autón. México 13: 185-242.

Amezcua-Linares, F. 1977. Generalidades ictiológicas del sistema lagunar costero de Huizache-Caimanero, Sinaloa, México. An. Centro. Cien. del Mar y Limnol. Univ. Nal. Autón. México 4: 1-26.

Amezcua-Linares, F. 1990. Los peces demersales de la plataforma continental del Pacífico central de México. Tesis de Doctorado en Ciencias del Mar, Universidad Nacional Autónoma de México, México.

Anónimo. 1980. Estudio oceanográfico del Golfo de Tehuantepec. Tomos I-III (Biología; Física y Química; 
Geología). Secretaría de Marina, Dirección General de Oceanografía, Talleres de la Nación, México.

Castro-Aguirre, J.L. 1978. Catálogo sistemático de los peces marinos que penetran a las aguas continentales de México con aspectos zoogeográficos y ecológicos. Instituto Nacional de la Pesca. Depto. de Pesca, México. 298 p.

Castro-Aguirre, J.L. 1982. Los peces de las Lagunas Oriental y Occidental, Oaxaca, México y sus relaciones con la temperatura y salinidad. II Análisis Multifactorial. Anales de la Escuela Nacional de Ciencias Biológicas, México 26: 85-100.

Castro-Aguirre, J.L., M.J. Parra-Alcocer \& F. de LachicaBonilla. 1974. Los peces de las Lagunas Oriental y Occidental, Oaxaca, y sus relaciones con la temperatura y salinidad. Mem. V Congreso Nacional de Oceanografía, Guaymas, Sonora, México: 148-161.

Cervantes-Castro, D. 1969. Estabilidad del acceso a la Laguna del Mar Muerto, Chiapas, México, p. 367-376. In A. Ayala-Castañares \& F.B. Phleger (eds.). Lagunas Costeras un Simposio. Memorias del Simposio Internacional Lagunas Costeras. UNAM-UNESCO, Nov. 28-30, 1967, México.

Chávez, E.A. 1979. Análisis de la comunidad de una laguna costera en la costa sur occidental de México. An. Centro. Cien. del Mar y Limnol. Univ. Nal. Autón. México 6: 15-44.

Greenwood, P.H., D.E. Rosen, S.H. Weitzman \& G.S. Myers. 1966. Phyletic studies of teleostean fishes, with a provisional classification of the living forms. Bull. Amer. Mus. Nat. Hist. 131: 341-455.

Jordan, D.S. \& B.W. Evermann. 1896-1900. The fishes of North and Middle America. Bull. U.S. Nat. Mus. 1-4: $1-3313$

Meek, E.S. 1902-1904. The fresh-water fishes of Mexico north of the Isthmus of Tehuantepec. Fiel. Colum. Mus. Zool. Ser. 5: 1-252.

Meek, E.S. \& S.F. Hildebrand. 1923-1928. The marine fishes of Panama. Field. Mus. Nat. Hist. Publ. Zool. Ser. 15: 1-1045.

Nelson, J. S. 1994. Fishes of the world, Wiley, Nueva York. $600 \mathrm{p}$.

Tapia-García, M., M.C. García-Abad, G. González-Medina, M.C. Macuitl-Montes \& G. Cerdenares L. de Guevara. 1994. Composición, distribución y abundancia de la comunidad de peces demersales del Golfo de Tehuantepec, México. Trop. Ecol. 35: 229-252.

Yáñez-Arancibia, A. 1978. Taxonomía, ecología y estructura de las comunidades de peces en lagunas costeras con bocas efímeras del Pacífico de México. Centro Cien. del Mar y Limnol. Univ. Nal. Autón. México, Publ. Esp. 2: 1-306.

Yáñez-Arancibia, A.1986. Ecología de la zona costera: análisis de siete tópicos. AGT, México. 189p. 